\title{
MERGING GROUND-BASED AND SPACEBORNE INSAR DATA TO MONITOR AN EARTH DAM
}

\author{
Luigi Mascolo (1), Giovanni Nico (2), Alfredo Pitullo (3) \\ (1) DIAN s.r.l., 75100 Matera, Italy,Email:1.mascolo@dianalysis.eu \\ (2) CNR-IAC, 70126 Bari, Italy, Email: g.nico@ba.iac.cnr.it \\ (3) Consorzio per la Bonifica della Capitanata, 71121 Foggia, Italy
}

\begin{abstract}
Monitoring of old embankment dams is currently carried out by installing devices that are able to provide just local measures in specific points of the structures. In this work, we propose the joint use of Ground-Based (GB) Synthetic Aperture Radar (SAR) and of spaceborne SAR interferometry to monitor the structural changes occurring on earth-fill or rock-fill dams. Measurements obtained by such techniques can be exploited to obtain three-dimensional maps of displacements with sub-millimeter precision in a noninvasive way. The results of monitoring campaigns of two earth-fill dams are presented.
\end{abstract}

\section{INTRODUCTION}

Synthetic Aperture Radar (SAR) data from spaceborne sensors are regularly acquired with revisiting times ranging from about 40 days to less than 11 days and are characterized by high spatial resolution (from more than $20 \mathrm{~m}$ to less than $3 \mathrm{~m}$ ). Spaceborne SAR interferometry has proved a mature technique in a number of applications, such as the monitoring of geological phenomena, infrastructures and urban areas [1,2].

Recently, the use of GB-SAR sensors for the monitoring of landslides and buildings has been demonstrated and studies about the mitigation of atmospheric effects, on the use of polarimetric sensors and on focusing techniques GB-SAR have been presented [3]-[12]. Applications to the monitoring of landslides, bridges, dams and several man-made structures appear in [13]-[16].

New techniques are needed for the monitoring of dams, which are mostly still equipped with devices that are able to provide just local measures in specific points of the structure. In this work, the use of SAR interferometry is demonstrated for the monitoring of the structural changes affecting earth-fill or rock-fill dams.
Measurements obtained by such techniques provide overall maps of displacements with sub-millimeter precision in a non-invasive way. We propose a protocol for the monitoring of old embankments based on the joint use of spaceborne and Ground-Based SAR interferometry and demonstrate strategies of applications of such techniques. While spaceborne sensors are suitable for measuring vertical displacement vectors of dams, GB-SAR sensors can be used to obtain maps of the horizontal plane projection of the overall displacement vector. Experiments have been carried out by using SAR data acquired by a Ku-band GBSAR sensor and the X-band Cosmo-Sky-Med sensor.

The paper is organized as follows. In Section 2 we provide a general description of the SAR interferometry technique. Then, in Section 3 and 4, we present the results of both GBSAR and spaceborne SAR data analysis. The conclusions close the paper.

\section{SYNTHETIC APERTURE RADAR (SAR) INTERFEROMETRY}

A Synthetic Aperture Radar (SAR) is an active microwave sensor used to produce two-dimensional microwave images of the observed scene [1]. The main advantage of microwave images resides in the capability of microwaves to pass through clouds, allowing to capture a scene on the Earth surface in any weather condition and illumination condition. For these reasons, this SAR has been extensively used in spaceborne missions to observe the Earth surface.

SAR interferometry (InSAR) consists of the processing two coregistered SAR images of the same scene, obtained from either two different positions (spatial-baseline interferometry) or from the same position (zero-baseline interferometry). 


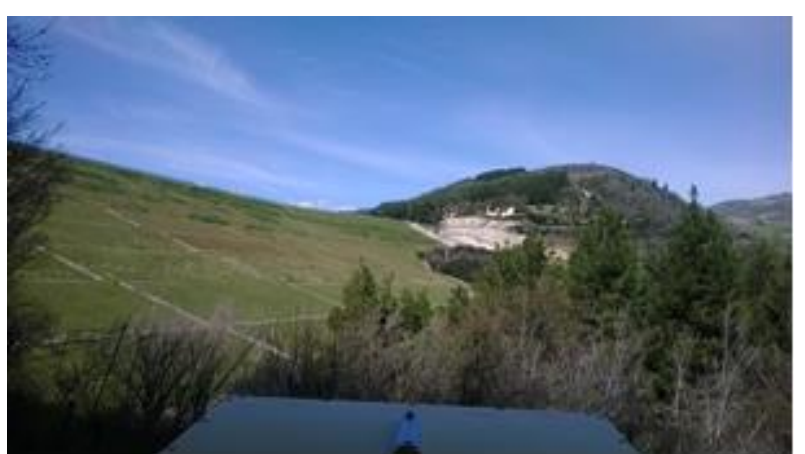

(a)

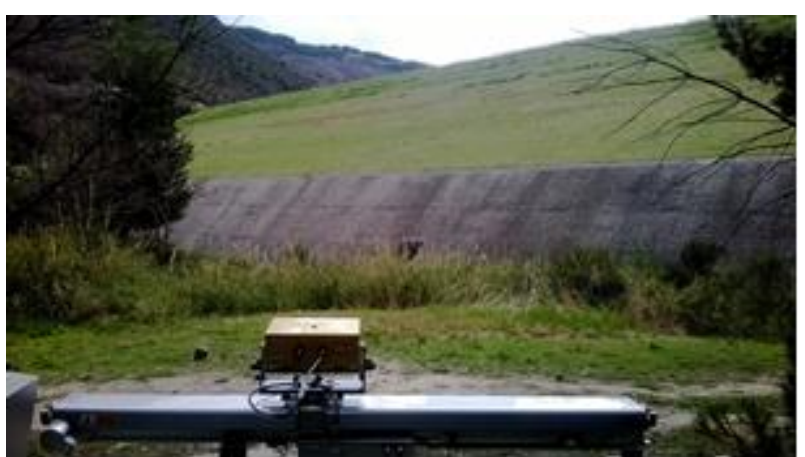

(b)

Figure 1: Downstream view of the Occhito dam. Figures (a) and (b) show the left and right side monitoring views.

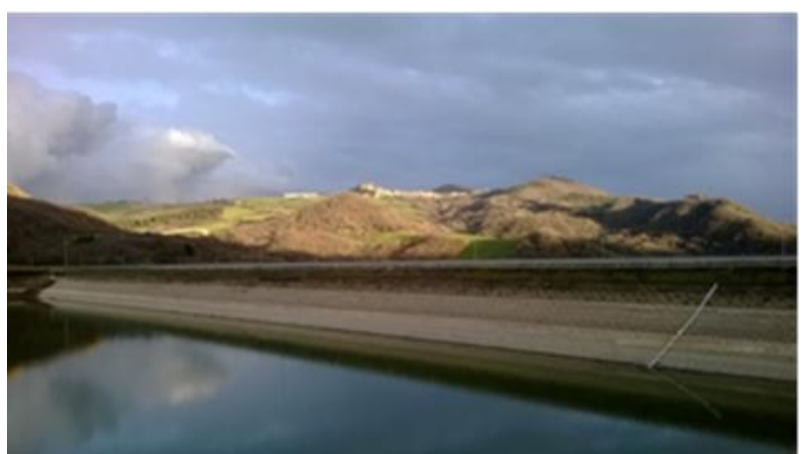

(a)

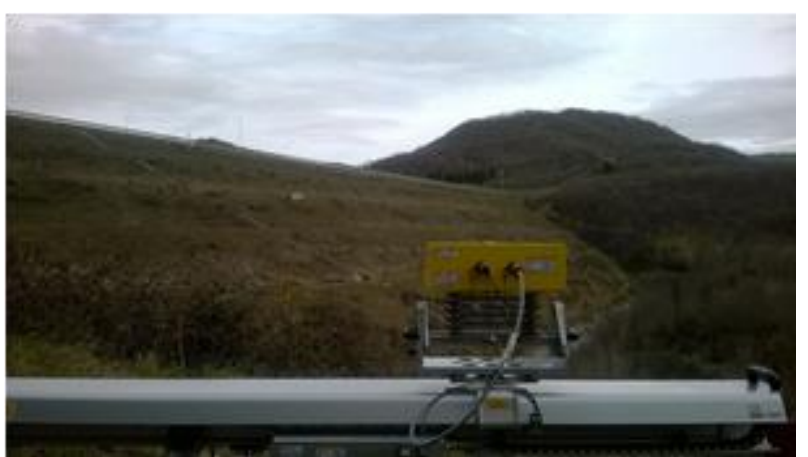

(b)

Figure 2: Views of the Osento dam. The upstream and the downstream monitoring views are shown in (a) and (b) respectively.

The first acquisition geometry is typical of repeat-pass spaceborne SAR interferometry, while the zero-baseline interferometry is typical of Ground-Based SAR interferometry.

A SAR image is a two-dimensional array of pixels carrying both the amplitude and the phase information of the reflected wave. Each pixel corresponds to a surface whose dimensions are very large compared to the radar wavelength. This surface contains a large number of elemental scatterers. The returned echo is the result of the coherent summation of all the returns due to the single scatterers. The echo power depends on the dielectric properties of scatterers, their spatial distribution and their orientation with respect to the SAR sensor. If the same surface is observed from two slightly different positions of the SAR sensor, the returned echo differs. Furthermore, the returned echo also changes over time due to modifications in the scene.

SAR interferometry uses differential phase of the reflected signal. By differencing the phases of two coherent complex-valued SAR images, it is possible to infer the phase contribution due to the scene morphology or caused by its displacement, in which case the phase difference is proportional to the displacement normalized to the radar wavelength.

Spaceborne SAR sensors observe the Earth surface with revisiting times ranging from a few days to a few weeks depending on the satellite carrier. The data they acquire can be processed using SAR interferometry techniques to provide information about displacement phenomena of terrain or structures. Using data of both current and past spaceborne SAR missions, it is possible to monitor current displacement and to study displacement phenomena occurred or started in the recent past.

One limitation of spaceborne SAR interferometry resides in that it provides just a projection of the displacement along the sensor line-of-sight (LOS). Therefore, it follows that a spaceborne sensor can detect only a very small component of horizontal component of the displacement vector, depending on the acquisition configuration. GB-SAR sensors provide a complementary information for the characterization of the real displacement vector.

A ground-based SAR system is a steppedfrequency radar. It is composed by a couple of transmitting/receiving antennas, which are mounted on a computer-controlled positioner that synthesizes a linear aperture along the azimuth direction. A microwave source illuminates the scene at different discrete frequencies.

One great advantage of GBSAR interferometry with respect to traditional monitoring techniques is its capability to provide areal information on the displacement field rather than measurements of displacements in only a few points. 


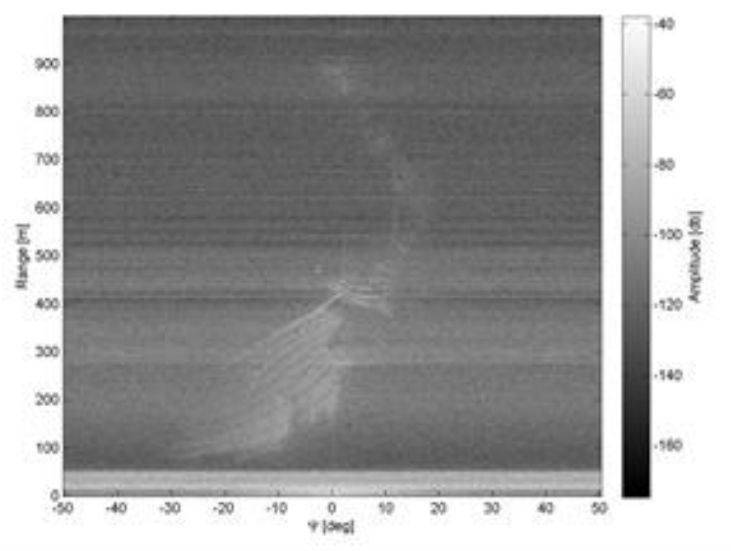

(a)

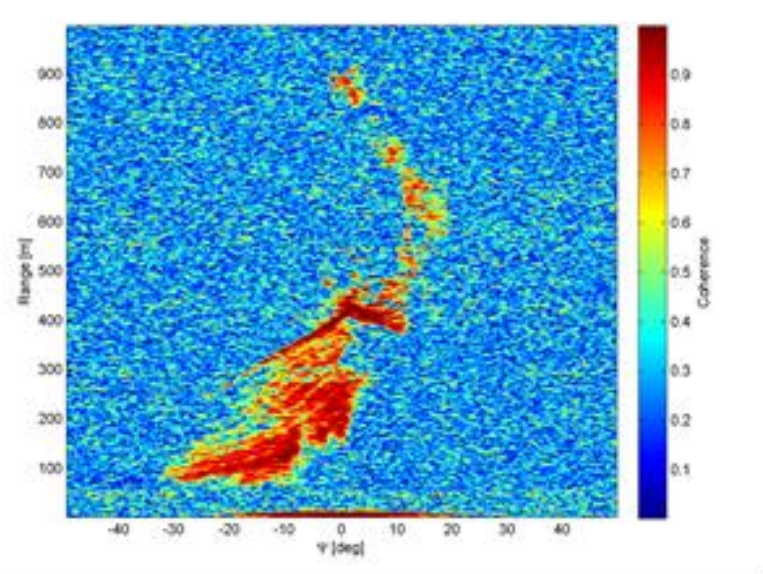

(c)

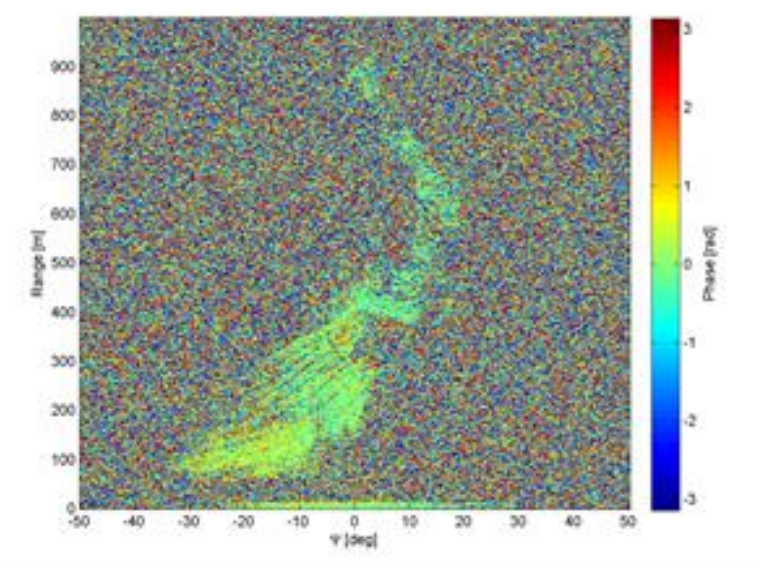

(e)

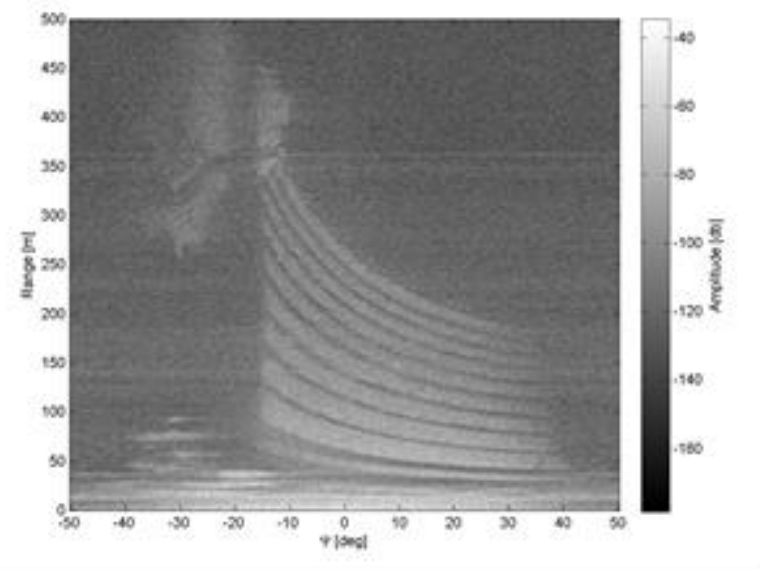

(b)

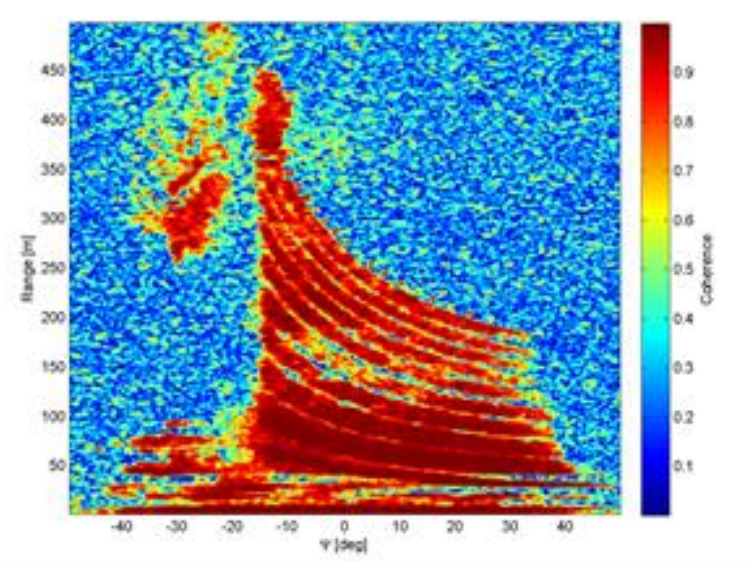

(d)

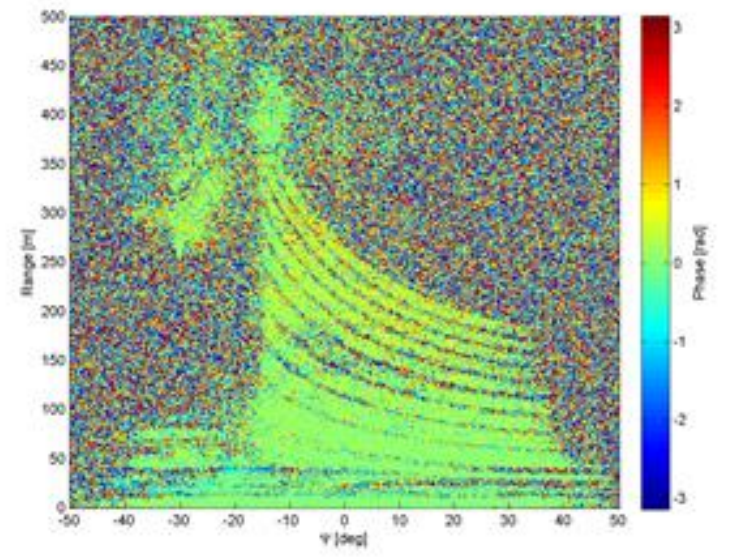

$(f)$

Figure 3: Interferometric analysis of GB-SAR data acquired over the area of the Occhito dam. Images in each column represent the amplitude maps of the scene $(a, b)$, the coherence maps $(c, d)$ and the interferometric phase maps $(e, f)$. The left column refer to the left side of the dam, while the right one to the right side (both from the downstream side). Images were acquired during the same day.

\section{RESULTS}

We present the results of monitoring campaigns carried out to demonstrate the application of Ground Based and
Spaceborne SAR sensors for the monitoring of earthfill or rockfill dams. Experiments have been carried out on two different earthfill dams, namely the Occhito and the Osento dams, which are located at the northern part of the Apulia Region, in the South of Italy, and managed 


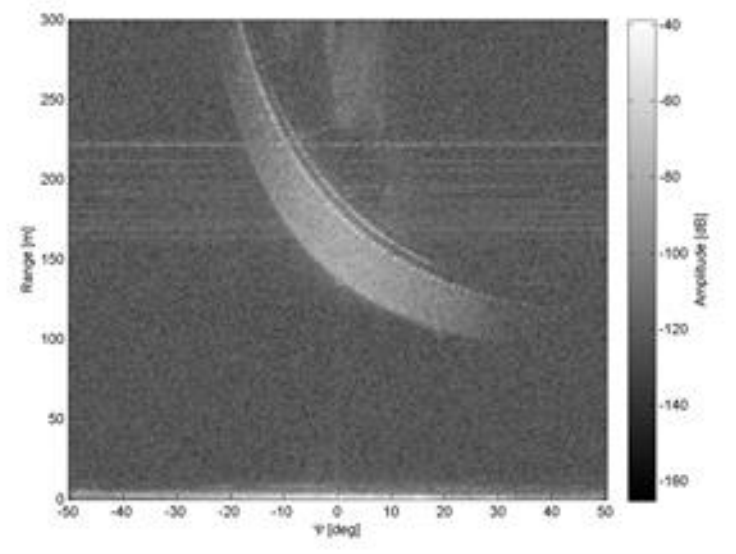

(a)

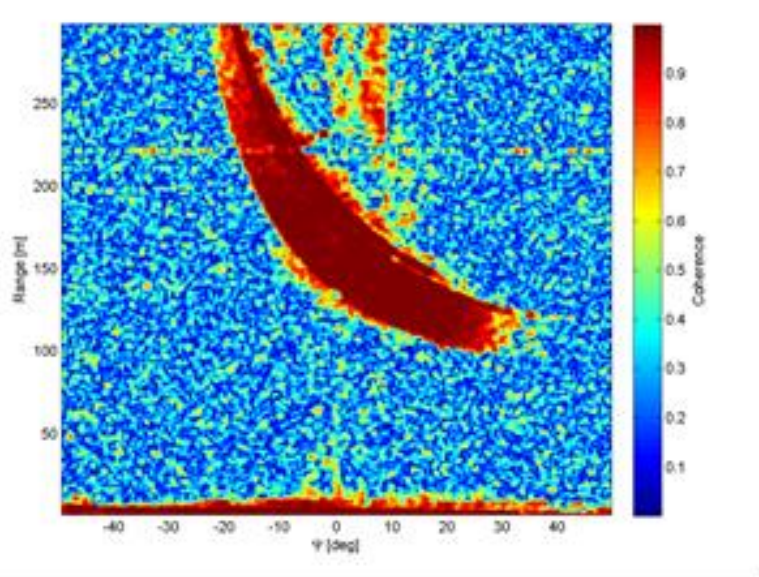

(c)

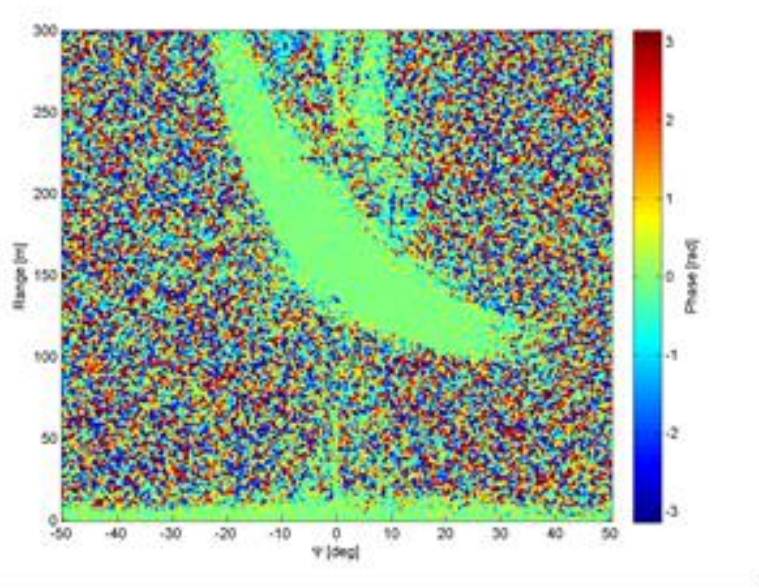

(e)

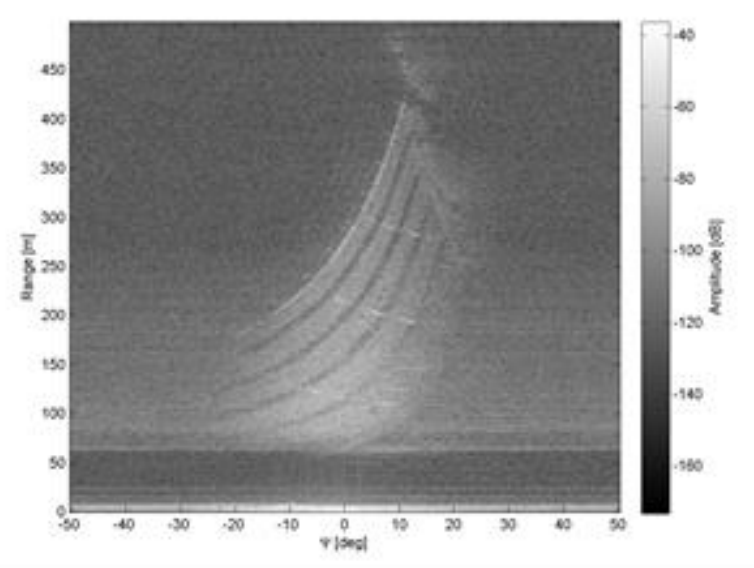

(b)

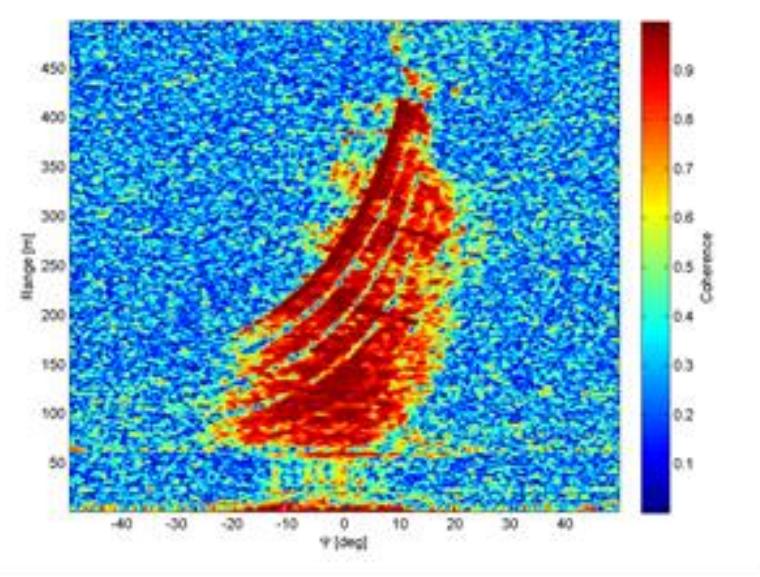

(d)

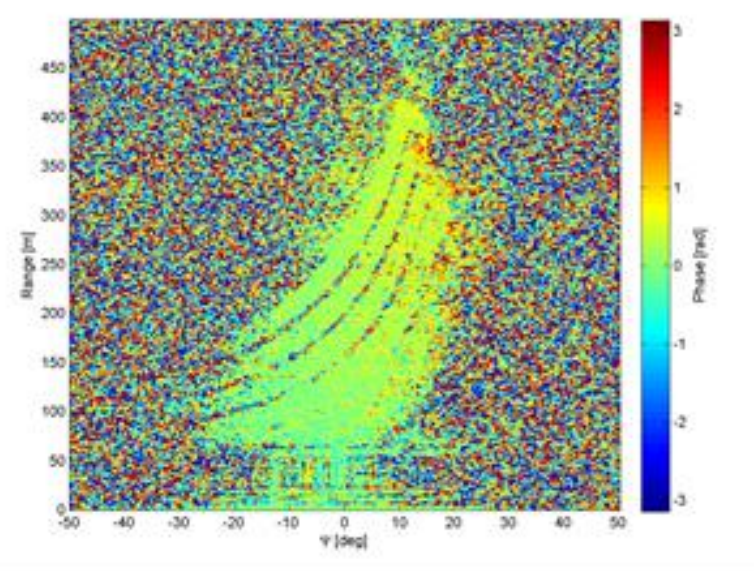

(f)

Figure 4: Interferometric analysis of GB-SAR data acquired over the area of the Osento dam. Images in each column represent the amplitude maps of the scene $(a, b)$, the coherence maps $(c, d)$ and the interferometric phase maps $(e, f)$. The left column refer to the upstream side of the dam, while the right one to the downstream side. Images were acquired during the same day.

by the Consorzio per la Bonifica della Capitanata.

\subsection{Ground-Based SAR monitoring}

Two experiments have been carried out in order to assess and validate the possibility to measure the displacements affecting a dam structure, due to water level variations. Such variations cause displacements of the dam structure in the up-stream to down-stream direction and mainly involve the central part of the dam. In this work, we used a Ku-band GB-SAR, with a 2 


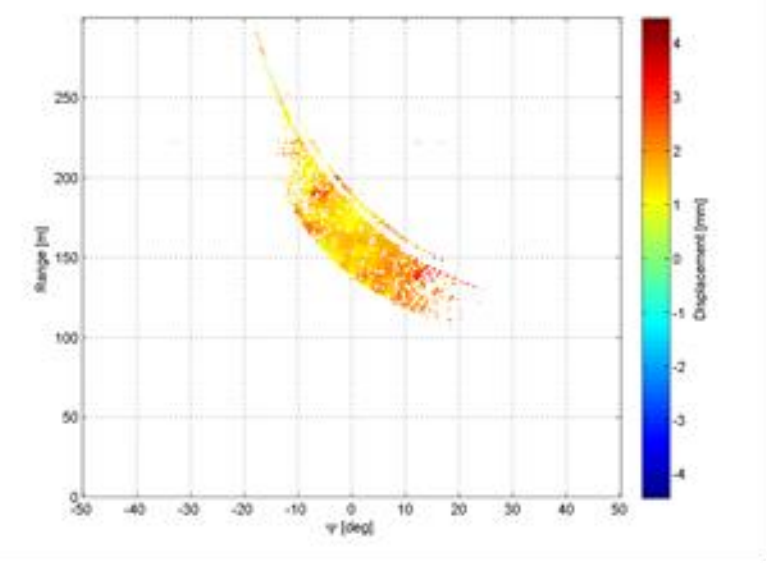

(a)

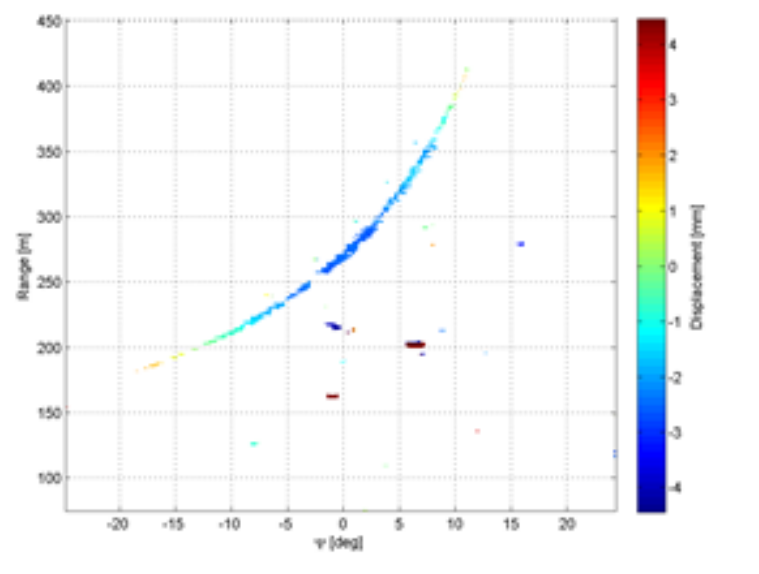

(b)

Figure 5: Maps of displacements of the Osento dam test site. (a) refers to the upstream side of the dam, (b) to the downstream side.

meters long synthetic aperture. Sampling rates in frequency and space are set in order to satisfy the Nyquist criterion. The working range distance to the study area ranges from about less than $100 \mathrm{~m}$ up to more than $7000 \mathrm{~m}$. The spatial resolution in range is $0.75 \mathrm{~m}$ while that in azimuth varies linearly with the distance between the radar and the observed scene from less than $1 \mathrm{~m}$ to a few meters. In each acquisition, the objects in the scene is mapped into a GB-SAR image in terms of range and azimuth distances, where the azimuth direction is set by the orientation of the rail.

The first experiment has been carried out on the Occhito dam. We have performed an interferometric analysis with a constant water level condition, i.e. with images acquired during the same day. Two monitoring sites have been chosen, at the downstream side, on the left and right-hand sides, as shown in Figure 1. The aim was to verify the possibility to capture the central section of the dam from both sites and therefore to combine the two LOS components. The results of this experiment are reported in Figure 3. For both monitoring sites, the amplitude, coherence and phase maps are shown. The amplitude images provide us an image of the structure of the dam as measured by the radar sensor from two different sides. The coherence maps are an indication of the precision of the radar measurement. Indeed, the stronger the coherence, the higher the quality of the measurement. Coherence depends on both the properties of the scene and the acquisition configuration. Areas covered by vegetation give rise to coherence losses due to random movements of leaves and branch

$\mathrm{s}$ and, therefore, displacement measurements are nosier [13]. Finally, interferometric phase maps are also shown, which can be translated to displacement maps, according to

$$
d=\frac{\lambda}{4 \pi} \Delta \phi
$$

where $\lambda$ is the radar wavelength.
From the images, it can be observed that an overlapping area exists between the left and right side dam measurements. It corresponds to the central part of the dam. The almost constant zero-phase value over the structure of the dam indicates that no displacement happened during the observation period, which is consistent to what expected in a condition of constant level of water.

The second experiment has been carried out on the Osento dam in both a constant and varied water level condition. In this case, monitoring sites have been positioned at the downstream side of the dam and at the upstream too, where a control platform was present. A photo of both sites is shown in Figure 2. The results of the experiments in condition of constant level of water are reported in Figure 4. Images were acquired during the same day. For this reason, high values of coherence resulted on both sites, due to no change of vegetation, for the downstream side, and to no change of water level for the upstream side. As expected, no displacements have been measured, as it can be inferred from the interferometric phase maps. In Figure 5 we report the displacement maps obtained in the condition of varied water level. For this experiment, measurements were performed with a temporal baseline of about 70 days, during which the water level increased after a rainy season. The displacement maps have been masked in order to preserve measures for areas characterized by strong coherence and amplitude values only. Losses of coherence happened at both sites, due to the presence of water, for the upstream side, and to the presence of vegetation, for the downstream side. In both cases, the crown of the dam presented strong coherence between the two dates. Measures of displacement are consistent with the change in the water level. Negative displacements in the maps mean a movement toward the radar position and viceversa for positive displacements. A $2 \mathrm{~mm}$ upstream-to-downstream movement was registered from both sites with respect to the central part 


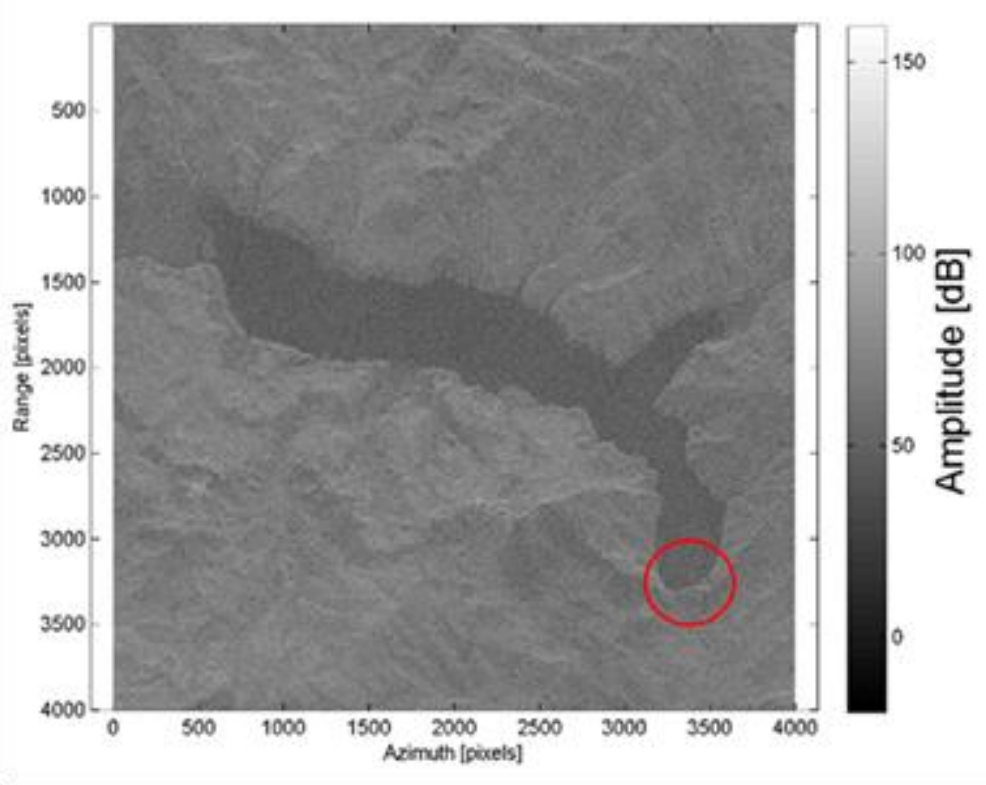

(a)

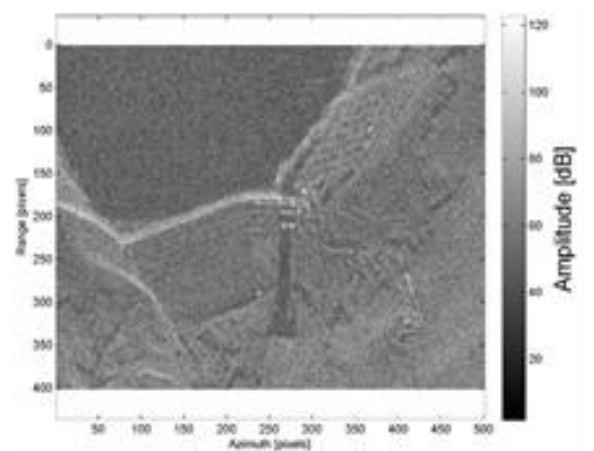

(b)

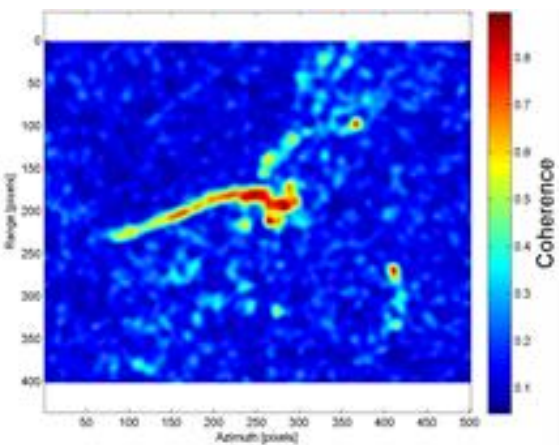

(c)

Figure 6: Cosmo-Sky-Med SAR data acquired over the Occhito lake test site. The SAR amplitude map of the scene is shown in $(a)$, where the red circle indicates the dam; images $(b)$ and $(c)$ report the dam amplitude and coherence maps respectively.

of the dam. Higher displacements, were measured in correspondence of concrete structures that were present on the dam surface, as observed from the downstream side.

The results presented for the two experiments demonstrate that a useful protocol for the monitoring of a dam consists of choosing monitoring sites on both the upstream and downstream sites and on the left and right sides of the dam. Combining such measurements, an estimate of the horizontal component of the displacement vector can be used to monitor the dam behaviour with strong accuracy.

\subsection{Spaceborne SAR monitoring}

Apart from horizontal plane displacements, dams can be affected also by subsidences, and therefore by vertical displacements. From this point of view, GBSAR are not suitable, as they can measure only a small component of the vertical displacement. In contrast, spaceborne SAR are more appropriate in this context.

We present the results obtained by processing data acquired by the Cosmo-Sky-Med sensor over the area of the Occhito lake. Images from Cosmo-Sky-Med SAR sensor have a 16 days temporal baseline and a $3 \mathrm{~m}$ spatial resolution. We here aim at verifying the possibility to capture the geometrical structure of the dam, which is surrounded by dense vegetation.

Two images are analysed, characterized by a temporal baseline of about one year and a half. In Figure 6 (a), the amplitude image of the area over the Occhito lake is illustrated. The red circle in the image indicates the location of the dam. For the circled area, we report the amplitude and the interferometric coherence maps evaluated on the two available images, in Figure 6 (b) and (c) respectively. The dam structure is well identified by the amplitude image. Furthermore, we observe that the crown of the dam is characterized by strong coherence, in consistence with the results obtained with the GB-SAR, while the surrounding area (water or vegetation) is characterized by low signal-tonoise ratios.

These first results suggest that earthfill embankment dam can be well identified in spaceborne SAR images and, therefore, a complete protocol for dam monitoring would consist of combining geolocated data from both GB- and spaceborne SAR, in order to obtain an overall displacement vector by using the interferometry technique.

\section{CONCLUSIONS}

In this work, we have presented a possible protocol for the monitoring of old embankment dams by using the SAR interferometry technique. The application of GBSAR interferometry for the monitoring of dam displacements has been demonstrated. The technique allows obtaining accurate two-dimensional maps of 
displacement. The results obtained from different observation sites can be combined in order to obtain an estimate of the horizontal plane component of the displacement vector. Furthermore, the first results of the use of spaceborne SAR interferometry have been shown, suggesting that merging spaceborne and GBSAR measures is a promising way to measure overall three-dimensional displacement vectors on large areas.

\section{REFERENCES}

[1] 1 D. Massonnet, K.L. Feigl, "Radar interferometry and its application to changes in the earth's surface", Reviews of Geophysics, 36(4), 441-500, (1998)

[2] G. Fornaro, F. Lombardini, A. Pauciullo, D. Reale, F. Viviani, "Tomographic processing of interferometric SAR data", IEEE Signal Processing Magazine, 31(4), 41-50, (2014).

[3] D. Leva, G. Nico, D. Tarchi, J. FortunyGuasch, A.J. Sieber, "Temporal analysis of a landslide by means of a ground-based SAR interferometer", IEEE Transactions on Geoscience and Remote Sensing, vol. 41(4), 745-752, (2003).

[4] M. Pieraccini, G. Luzi, D. Mecatti, M. Fratini, L. Noferini, L. Carissimi, G. Franchioni, C. Atzeni, "Remote sensing of building structural displacements using a microwave interferometer with imaging capability", NDT \& E International, vol. 37, 545 - 550, (2004).

[5] G. Antonello, N. Casagli, P. Farina, D. Leva, G. Nico, A.J. Sieber, D. Tarchi, "Ground-based SAR interferometry for monitoring mass movements", Landslides, vol. 1(1), pp. $21-28$, (2004).

[6] G. Nico, D. Leva, J. Fortuny-Guasch, G. Antonello, D. Tarchi, "Generation of digital terrain models with a ground-based SAR system", IEEE Transactions on Geoscience and Remote Sensing, vol. 43(1), 45 - 49, (2005).

[7] L. Pipia, X. Fabregas, A. Aguasca, C.LopezMartìnez, "Atmospheric artifact compensation in ground-based DInSAR", IEEE Geoscience and Remote Sensing Letters, 5(1), 88-92, 2008.

[8] L. Pipia, X. Fabregas, A. Aguasca, C. LopezMartìnez, S. Duque, J.J. Mallorquì, J. Marturìa, "Polarimetric differential SAR interefrometry: first ersults with ground-based measurements", IEEE Geoscience and Remote Sensing Letters, vol. 6(1), pp. 167 - 171, (2009).

[9] L. Pipia, X. Fabregas, A. Aguasca, C. LopezMartìnez, "Polarimetric temporal analysis of urban environments with a ground-based SAR", IEEE Geoscience and Remote Sensing Letters, vol. 51(4), pp. 2343 - 2360, (2013).
[10] Fortuny-Guasch, J., "A fast and accurate far field pseudo - polar format radar imaging algorithm”, IEEE Transaction on Geoscience and Remote Sensing, vol. 47, no. 4, 11871196, (2009).

[11] Monti Guarnieri, A and Scirpoli, S., "Efficient wavenumber domain focusing for groundbased SAR, IEEE Geoscience and Remote Sensing Letters, vol. 7, no. 1, 161-165, (2010).

[12] Guccione, P., Zonno, M., Mascolo, L., and G. Nico, "Focusing algorithms analysis for ground-based SAR images", Proceeding of the International Geoscience and Remote Sensing Symposium (IGARSS), 3895 - 3898, (2013).

[13] A. Di Pasquale, M. Corsetti, P. Guccione, A. Lugli, M. Nicoletti, G. Nico, M. Zonno, "Ground-based SAR interferometry as a supporting tool in natural and man-made disasters", In: Proceedings of the 33rd EARSel Symposium,173-186, Lasaponara R., Masini N., Biscione M. Editors, (2013).

[14] G. Nico, L. Borrelli, A. Di Pasquale, L. Antronico, G. Gulla, "Monitoring of an ancient landslide by GBSAR technique in the Maierato town (Calabria, Italy)", Engineering Geology for Society and Territory, 1-5, vol.2, Springer International Publishing International, ISBN 978-3-319-09056-6, (2014).

[15]G. Nico, M. Corsetti, A. Di Pasquale, D. Donnarumma, L. Dotti, L. Fiorentino, M. Nicoletti, "On the monitoring of dams by means of ground-based radar interferometry (GB-SAR)", $9^{\text {th }}$ ICOLD European Club Symposium Proceedings 2013 (in press).

[16] Luigi Mascolo, Giovanni Nico, Andrea Di Pasquale, Alfredo Pitullo, "Use of advanced SAR monitoring techniques for the assessment of the behaviour of old embankment dams", Proceedings of SPIE Vol. 9245, 92450N (2014)

[17] G. Nico, R. Tome, J. Catalao, P.M.A. Miranda, "On the use of the WRF model to mitigate tropospheric phase delay effects in SAR interferograms", IEEE Transactions on Geoscience and Remote Sensing, 49(12), 49704976, (2011).

[18]P. Mateus, G. Nico, R. Tome, J. Catalao, P.M.A. Miranda, "Experimental study on the atmospheric delay based on GPS, SAR interferometry, and numerical weather model data", IEEE Transactions on Geoscience and Remote Sensing, 51(1), 6-11, (2013). 